\title{
LA DIÁSPORA DE PROFESIONALES COMO ACTIVO PARA LA INTERNACIONALIZACIÓN DE LA ECONOMÍA
}

El objetivo del artículo es analizar el papel que puede desempeñar la diáspora de profesionales como un activo para la internacionalización de una economía. Se presta una especial referencia al caso de España, que ha registrado a partir de la crisis económica que se inició en 2008 un fuerte proceso de marcha de profesionales al extranjero, sobre todo jóvenes. Se identifican una serie de canales a través de los cuales esta diáspora puede contribuir a la internacionalización de la economía y las empresas. Las Administraciones públicas deben prestar atención al fenómeno de la diáspora y diseñar estrategias y acciones que faciliten su aprovechamiento.

\section{The professional diaspora as an asset for the internationalization of the economy}

The objective of the article is to analyze the role that the diaspora professionals can play as an asset for the internationalization of economies. A special reference is made to the case of Spain, which has registered a strong growth of professionals abroad, especially young people, since the economic crisis that began in 2008. A series of channels are identified through which this diaspora can contribute to the internationalization of an economy and its companies. Governments must pay attention to the diaspora phenomenon and design strategies and actions that facilitate its use.

Palabras clave: talento, emigración, políticas de internacionalización.

Keywords: talent, migration, internationalization policies.

JEL: F22, F23.

1. Introducción

En las últimas décadas se ha desarrollado una importante literatura en economía que analiza el impacto de las poblaciones emigrantes (o de origen emigrante) tanto en sus países de residencia como en sus países de origen. El fuerte aumento de los flujos

\footnotetext{
* Técnico Comercial y Economista del Estado.

Socio Fundador de Iberglobal Consultoría de Internacionalización.

Versión de marzo de 2020

DOI: https://doi.org/10.32796/ice.2020.913.6989
}

migratorios, la creciente atención a la emigración como una cuestión de gran relevancia social, económica y política, se encuentran detrás de la atención a este tema. En época más reciente se ha empezado a prestar atención a lo que puede denominarse diáspora profesional, es decir, la diáspora que se genera a partir de la emigración de personas de un nivel profesional y educativo relativamente alto.

Hasta hace relativamente poco tiempo, gran parte de la literatura se ha dedicado a países en desarrollo, y al impacto económico que tiene sobre ellos el disponer de una diáspora en otros países. El tema de 
la emigración de profesionales cualificados desde países avanzados era relativamente poco estudiado.

El objeto de este artículo no es la diáspora o la emigración en general, sino la diáspora profesional, que se caracteriza por un nivel relativamente alto de cualificación. De manera más concreta, nuestro objetivo es analizar el papel que esta diáspora profesional puede desarrollar como activo para la internacionalización de las economías de sus países de origen.

En los primeros dos apartados trataremos del ascenso en general de la diáspora profesional y del cambio que ha habido en su percepción, hacia una percepción más positiva. En los dos siguientes apartados analizaremos cuáles son las principales ventajas que puede suponer la diáspora: en primer lugar, las ventajas para los propios profesionales que salen al exterior; $y$, en segundo lugar, las ventajas que la diáspora puede aportar a los países de origen de estos profesionales. En el siguiente apartado analizaremos los motivos que puede tener la diáspora para participar en iniciativas dirigidas hacia ella. En el último apartado analizaremos el papel que pueden desempeñar las Administraciones públicas en la instrumentación de iniciativas encaminadas a identificar y aprovechar el activo de la diáspora.

\section{El desarrollo de una nueva diáspora profesional}

Se está prestando una creciente atención en numerosos países al papel que sus diásporas establecidas en otros países del mundo pueden desempeñar para favorecer su crecimiento económico.

Refiriéndonos a la etapa contemporánea de las últimas décadas, en una primera fase la mayor atención se prestó a las diásporas de países en vías de desarrollo (países asiáticos como India, China y Filipinas, países africanos, países latinoamericanos, etcétera) hacia países desarrollados, y que comprendían tanto personas de baja como de alta cualificación profesional. Quizás el caso más destacado, por su relevancia numérica y su extensión por diversas zonas del mundo, ha sido el de la diáspora china, que ha desempeñado un papel muy relevante en el proceso de reforma y apertura al exterior que China emprendió a partir de fines de los años setenta del siglo pasado.

La emigración de personas de alta cualificación (entendiendo por tales aquellas que han completado una educación superior) ha sido tradicionalmente importante en los países en desarrollo, con tasas de emigración anuales que pueden oscilar entre el $0,6 \%$ de Brasil y el $15 \%$ en Uganda, según Richard Devane (Devane, 2006). Como destaca este autor, Estados Unidos es el principal destino de emigración para trabajadores cualificados: el $40 \%$ de su población adulta nacida en el extranjero tiene educación superior. Estados Unidos también recibe el $32 \%$ de todos los estudiantes extranjeros que estudian en países de la OCDE. La educación superior es de hecho un importante canal para el reclutamiento por parte de empresas de Estados Unidos de personal de alta cualificación. Según señala Devane en su artículo, de las start-ups de Silicon Valley un cuarto de ellas tenía ejecutivos que eran de etnia china o india.

La literatura económica ha estudiado de manera amplia el impacto económico de la emigración. Este impacto se centra, en primer lugar, en las remesas que envían los emigrantes a sus países de origen, que oscilan en torno a 500.000 millones de dólares al año (Hausmann, 2015). En segundo lugar, se ha analizado el impacto que tienen las inversiones que realizan estas diásporas en sus países de origen.

Pero en los últimos 15 o 20 años ha aumentado la relevancia de un nuevo tipo de diáspora, que es la que interesa a los efectos del presente artículo. Se trata de una diáspora que tiene las siguientes características básicas:

- En primer lugar, los países originarios son países avanzados. Los países de destino, en los que se establecen estas diásporas, pueden ser tanto países avanzados como países emergentes.

- En segundo lugar, los movimientos migratorios de este período reciente han afectado no solo a trabajadores poco cualificados sino también a trabajadores 
de una cualificación relativamente elevada. Se puede hablar así del desarrollo de una diáspora profesional con una cualificación alta.

Detrás del desarrollo y crecimiento de esta diáspora profesional se encuentran diversos factores:

- En primer lugar, el gran impulso que ha recibido la globalización. En las últimas décadas, y favorecida por el avance tecnológico en los transportes y en las comunicaciones, así como por la liberalización de los intercambios económicos gracias a los procesos de liberalización impulsados por la Organización Mundial de Comercio (OMC), así como por los múltiples acuerdos regionales de comercio que han sido establecidos, la globalización ha experimentado un desarrollo extraordinario. Los crecientes movimientos de bienes y servicios han ido acompañados a su vez de un creciente movimiento de personas.

En el caso de las empresas españolas, su internacionalización ha contribuido a que crezca de forma sostenida el número de profesionales españoles establecidos en otros países. La exportación española ha continuado creciendo a buen ritmo en los últimos años, a pesar de la recuperación de la demanda interna, lo cual constituye un positivo indicador de que las empresas españolas han asumido que la internacionalización no es una actividad para compensar una desaceleración en el mercado doméstico, sino que es un requisito de competitividad al que se debe prestar la debida atención de forma permanente.

Por otra parte, muchos profesionales españoles son contratados por empresas internacionales y trabajan en otros países. Se marchan no por la crisis y la falta de trabajo en España, sino por la consecuencia lógica de la globalización del talento, que ha llevado a que las plantillas de las empresas estén formadas por profesionales de orígenes nacionales crecientemente diversos.

- En segundo lugar, la diversidad (cultural, nacional, racial) del talento ha pasado a ser considerada así como algo positivo, en contraposición con planteamientos del pasado en los que se consideraba que la diversidad era en cierta medida una especie de mal necesario o inevitable, o simplemente se ignoraba el papel que podía desempeñar en la eficiencia y capacidad de innovación de una organización.

La diversidad cultural de la mano de obra puede suponer ventajas u oportunidades. Dos aspectos pueden mencionarse a este respecto:

1) La diversidad puede fomentar la innovación. Una atmósfera de trabajo caracterizada por la diversidad cultural favorece la diversidad de puntos de vista y de enfoques, enriquece el análisis y la discusión, y en este sentido puede ser un factor que impulsa la innovación.

Algunos estudios han llegado a la conclusión de que la diversidad cultural es un factor que favorece la innovación. Es el caso, por ejemplo, del trabajo de Annekatrin Niebuhr, que estudió el impacto regional de la diversidad sobre la innovación en Alemania, midiendo por ejemplo el impacto que tiene la diversidad cultural en la producción de patentes per cápita entre las diferentes regiones alemanas, que era mayor en las regiones con mayor porcentaje de inmigrantes. Niebuhr realiza en su trabajo un repaso de algunos estudios sobre el tema, que concluyen que la diversidad cultural conduce hacia la creatividad y la innovación ya que implica una variedad en conocimientos y habilidades. "La heterogeneidad de la gente es importante para la creación de nuevas ideas» (Niebuhr, 2006).

La conclusión de Niebuhr es que «la diversidad cultural podría ser importante para la actividad de innovación a nivel regional. La evidencia empírica apunta a diferencias en el conocimiento y capacidades de los trabajadores de diversas culturas que pueden mejorar el rendimiento de I+D a nivel regional. Los beneficios de la diversidad parecen ser mayores que los efectos negativos».

2) Una mano de obra diversa favorece la internacionalización de la empresa y la economía en general, en la medida que facilita la superación de los problemas que pueden generar las barreras culturales e idiomáticas.

Este aspecto tiene una gran importancia y es relevante tanto a nivel de las empresas como de los países. Efectivamente, se puede considerar que países 
con un elevado grado de diversidad cultural (como Estados Unidos, Canadá o Australia) tienen una mayor ventaja para la actividad internacional de sus empresas al disponer de mano de obra preparada para trabajar en ambientes culturales diversos. Por ejemplo, contar con poblaciones de origen chino ha facilitado el trabajo en el mercado chino a las empresas de Australia y Estados Unidos, en comparación con las empresas de otros países que no contaban con poblaciones significativas de origen chino.

En resumen, los obstáculos que puede representar la diversidad en la plantilla de una empresa pueden ser ampliamente superados por las ventajas. Como señala un estudio realizado en España por la Fundación BBVA y el IESE sobre las políticas y prácticas de recursos humanos en las empresas multinacionales, «muchas empresas, fundamentalmente MNC (multinacionales), tanto en Estados Unidos como en la Unión Europea, parten de la base de que la diversidad es una fuente de ventajas competitivas. Estas ventajas se deben al hecho de que la diversidad bien dirigida conduce a una mejor comprensión de los mercados locales y clientes, a facilitar la resolución de problemas y a obtener una gran flexibilidad y, por consiguiente, una mayor productividad» (Quintanilla, Sánchez-Mangas y Susaeta, 2010).

El reconocimiento de la diversidad como factor positivo, y su promoción activa como parte de las estrategias corporativas, es un fenómeno reciente, y en el que las empresas españolas y latinoamericanas se hallan todavía retrasadas en comparación con otros países, como Estados Unidos y Gran Bretaña. El estudio que estamos citando de la Fundación BBVA-IESE considera «tres estadios, no excluyentes pero sí sucesivos. En un primer estadio, la empresa puede considerar la diversidad como el reconocimiento de que cualquier discriminación en base al sexo, cultura, raza, ideología, etc., es injusta. Un segundo estadio estaría formado por aquellas empresas que son conscientes del valor de la diversidad y la consideran algo positivo. Por último, aquellas que, por considerarla una fuente de ventaja competitiva, la promueven a través de sus políticas de selección, promoción profesional, etc.».

Una implicación de la positiva valoración de la diversidad es la creciente tendencia de las empresas multinacionales a situar centros de investigación y desarrollo en otros países distintos al de su origen o sede central. Ello conlleva con frecuencia el desplazamiento de investigadores de otros países a estos centros, aumentando así la diáspora profesional.

Hay empresas que, partiendo de la asunción de las ventajas de la diversidad, han establecido programas de rotación de personal entre los diferentes países en las que están establecidas. De esta forma intentan enriquecer el funcionamiento de sus equipos de trabajo, mediante la combinación de perspectivas diversas. Algunos estudios han demostrado que equipos de trabajo heterogéneos resolvían tareas complejas de mejor forma que equipos homogéneos. Esta estrategia corporativa también contribuye a potenciar la diáspora profesional. En tercer lugar, la diáspora de profesionales se ha visto impulsada por la Gran Recesión que se inició en 2008, que ha propiciado el que en una serie de países muchos profesionales emigraran en busca de oportunidades laborales, en mercados con mejores condiciones. Esta emigración ha sido especialmente notable en los países del sur de Europa, que se vieron más afectados por la crisis. De esta manera, se produjo una importante diáspora de profesionales españoles, buena parte de los cuales eran jóvenes, que se vieron impulsados a buscar trabajo en otros países debido al impacto de la crisis en España.

Con el tiempo, otro factor que ha impulsado esta diáspora no tiene que ver con las dificultades para encontrar empleo en España, sino con el deseo de desarrollar las capacidades profesionales a través de la experiencia en otras culturas y estilos de trabajo, acceder a mejores condiciones salariales y laborales.

En el caso de España la emigración hacia el extranjero de profesionales se canalizó mayoritariamente hacia países del norte de Europa, pero también en 


\section{ILUSTRACIÓN 1}

\section{Perfil de los expatriados españoles}

Datos demográficos de los españoles residentes en el extranjero
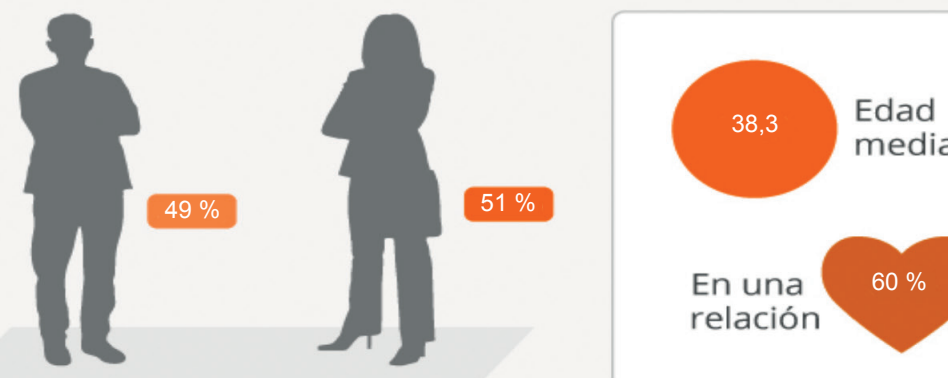

media

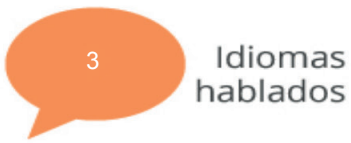

relación

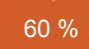

$60 \%$

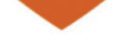

Estudios superiores*

Principales países de destino

Alemania

Reino Unido 粶

Holanda

Suiza
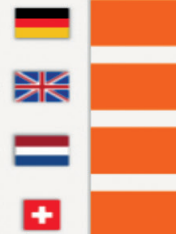

* Título de postgrado o máster

Fuente: InterNations statista

FUENTE: Statista, con datos de InterNations. https://es.statista.com/grafico/5791/como-son-los-expatriados-espanoles/

una apreciable medida hacia países de América (tanto Norteamérica como Latinoamérica) y otras zonas del mundo, en especial las economías asiáticas que han conocido en las últimas décadas un intenso crecimiento económico.

La última década ha contemplado por tanto un espectacular crecimiento de la diáspora de profesionales que van a trabajar y residir en países distintos a los suyos. Resulta difícil tener estadísticas más o menos precisas sobre este fenómeno, por varios motivos. En primer lugar, muchos emigrantes que se han instalado en otros países no se registran en el consulado de su país. Esto se aplica de manera especial a los países de la Unión Europea, entre los cuales existe libre circulación de personas y no hay un incentivo apreciable para llevar a cabo el registro consular.
Por otro lado, y de cara a estimar el alcance de la diáspora profesional, existe todavía una escasez de información estadística sobre el perfil laboral de los emigrantes.

A modo de ejemplo, en la llustración 1 se recoge el perfil de los expatriados españoles según un estudio de InterNations de 2016. Como puede verse, los países europeos son los principales destinos de los expatriados españoles, con Alemania en primer lugar, con un $12 \%$ del total, seguida del Reino Unido, Holanda y Suiza.

Hay que destacar el hecho de que un $57 \%$ de los expatriados, según este estudio, tienen estudios superiores. Igualmente los expatriados hablan una media de tres idiomas.

Estos datos, que deben ser considerados con prudencia por los motivos mencionados anteriormente, 
ponen de relieve una característica que intuitivamente parece clara, y que ya hemos apuntado anteriormente: el perfil de la emigración española a partir de la crisis de 2008 ha tenido un carácter marcadamente profesional, de personas con un nivel de cualificación relativamente elevado. Es una diferencia significativa con lo que fue la emigración española en épocas pasadas, en los años sesenta y setenta del siglo pasado, en los que la emigración canalizó fundamentalmente trabajadores poco cualificados.

\section{Visiones contrapuestas de la diáspora}

Existe una gran división en la forma de percibir los efectos de la diáspora por parte de la sociedad y de los políticos. Por un lado tenemos la visión negativa: se basa en la idea de que la marcha de profesionales al exterior supone una pérdida de talento para el país, hay que frenarla e incluso establecer medidas para incentivar su retorno. La otra visión es positiva: la marcha de profesionales a otros países tiene numerosos efectos positivos, tanto para estos profesionales como para sus países de origen.

En el caso de España, la marcha de profesionales al extranjero ha recibido con frecuencia una valoración negativa, tanto en medios de comunicación como por parte de actores políticos y otras instancias. Se ha hablado de que esta marcha suponía una «pérdida de talento» para España, puesto que el país "perdía» unos profesionales en cuya formación se habían invertido cuantiosos recursos, y que podían contribuir de manera efectiva a su desarrollo económico.

El falso punto de partida en este tipo de valoración es la idea de que la marcha de profesionales al extranjero supone una "pérdida de talento» para la economía española. No cabe hablar de pérdida de talento cuando la alternativa a la marcha al extranjero es quedarse en España en desempleo, sin tener ingresos, sin adquirir experiencia, sin perspectivas de progreso en una carrera profesional. No cabe hablar de pérdida de talento para la economía española cuando esta no tiene la capacidad de utilizar este talento. Las altas tasas de desempleo juvenil son la prueba de esta falta de capacidad.

Como ha señalado Javier Santiso (Santiso, 2012), «la mayor fuga de cerebros es la de informáticos y programadores que se quedan en el país y buscan sobrevivir con un oficio que no es el suyo; o bien se quedan en la casa familiar, esperando a que pase la tormenta, perdiendo año tras año conocimientos y habilidades al no ejercerlas».

Está claro que la marcha al extranjero puede tener aspectos negativos. En primer lugar, para muchos de los que se han ido no ha sido una opción voluntaria, sino que se vieron empujados a ello por la necesidad. En segundo lugar, España ha invertido efectivamente recursos en la formación de estos trabajadores; es una inversión desaprovechada, de la que se benefician en cambio los países que los reciben. En tercer lugar, parte de estos profesionales se han visto obligados a aceptar, en los países a los que emigraron, puestos por debajo de sus cualificaciones.

Sin duda, la situación más deseable es que estos profesionales tengan la opción de elegir si se quedan en España o si prefieren irse al extranjero. Pero no ha sido el caso, esa opción no ha existido. Para numerosas personas la única vía para encontrar un trabajo ha sido marcharse fuera. No cabe hablar por tanto de pérdida de talento para la economía española, puesto que esta se mostraba incapaz de ofrecer un empleo a ese talento.

La visión negativa ha llevado incluso a que en algunas Administraciones se hayan instrumentado programas para fomentar el «retorno del talento». De entrada, estos programas para incentivar el retorno pueden suponer una discriminación en contra de los trabajadores que están en España. Si una empresa tiene que contratar a alguien, y tiene un candidato que viene de fuera, que probablemente tiene un buen nivel de idiomas, una formación complementada por su experiencia en otros países y culturas, y además la empresa recibe una subvención por contratarle, pues 
la decisión que tomará probablemente será en favor de trabajadores «de fuera». Podría llegarse al caso en que las empresas, para contratar gente, recurrieran de forma sistemática a trabajadores «retornados», con el fin de beneficiarse de las ayudas públicas.

Este tipo de incentivos podría tener un efecto contrario al buscado: muchas personas podrían llegar a la conclusión de que tendrían que irse al extranjero para poder beneficiarse posteriormente de esos incentivos y mejorar sus perspectivas de contratación en España.

A lo expuesto en las páginas precedentes se añade el hecho de que España ha sido tradicionalmente una sociedad más cerrada, en comparación por ejemplo con otras sociedades europeas. Debido a una serie de factores históricos y culturales la sociedad española se replegó sobre sí misma durante el siglo XX. Los ciudadanos españoles mostraron poco interés por el mundo exterior, por viajar. Cristina Manzano explica estos condicionantes históricos de la tendencia al aislamiento en un artículo publicado en 2013 que tituló Es hora de que los españoles salgan de su aislamiento: «Desde principios del siglo XX, la sociedad española se encerró en sí misma. La pérdida definitiva del imperio colonial en el desastre del 98 fue el detonante que la sumió en un estado general de depresión y abatimiento, agravado por la constante convulsión política y por una deplorable situación económica. El país inició entonces un período de reflexión sobre el ser de una España que se replegaba al territorio de la península Ibérica, sus islas y unos pocos (e insignificantes) reductos en África y que se tradujo en un profundo ensimismamiento interno. La neutralidad durante la Primera Guerra mundial y, posteriormente, la guerra civil y el aislamiento internacional, voluntario y provocado, durante la dictadura franquista profundizaron esa brecha entre la sociedad española y el resto del mundo. Voluntario por la propia naturaleza del régimen; provocado porque la Segunda Guerra Mundial dividió a los países en aliados y eje, en vencedores y vencidos, en Este-Oeste; y España no estaba en ninguno de los dos lados. El exterior fue sospechoso durante casi 40 años» (Manzano, 2013).
Para Cristina Manzano, «Un elemento que sin duda ha influido también en nuestro ensimismamiento interior es la falta de una memoria colonial cercana, al contrario de lo que ocurre en otros países de nuestro entorno. Una buena parte de británicos, franceses, belgas o portugueses guardan referencias de primera o segunda generación de un pasado ligado a lugares exóticos y vidas diferentes, incluso aunque su pérdida supusiera un auténtico trauma nacional y humano. Proporcionalmente, el número de españoles que pasó por el protectorado español en Marruecos, por Guinea Ecuatorial o por el Sáhara fue muy inferior y su huella mucho menos profunda».

Otros factores contribuyen o se entremezclan con los mencionados en el párrafo anterior. Es bien conocida la escasa propensión de los españoles a la movilidad geográfica por cuestiones laborales, que en otras sociedades occidentales está mucho más extendida. Esta resistencia a la movilidad geográfica se manifiesta incluso cuando se trata de cambiar de residencia en España; la resistencia es mucho mayor si un nuevo empleo implica tener que desplazarse al extranjero. Según Manzano, «esta situación ha comenzado a cambiar significativamente por el agravamiento de la crisis. El Eurobarómetro de 2009 reflejaba que solo un 12 por cien de españoles tenía previsto salir del país por un empleo a lo largo de su vida laboral, frente a un 51 por cien de los daneses o un 38 por cien de los estonios» (Manzano, 2013).

A ello se añade una limitación tradicional de España, que es su fuerte déficit en lo que se refiere al conocimiento de lenguas extranjeras, una barrera que ha persistido tenazmente a lo largo del tiempo.

Frente a estos planteamientos negativos, las ventajas que la diáspora puede suponer para el desarrollo económico de un país están siendo reconocidas de manera cada vez más clara por los autores que estudian el tema. Por ejemplo, Newland y Plaza señalan que «más allá del envío de transferencias, (las diásporas) pueden promover el comercio y la inversión extranjera directa, crear negocios y promover el 
emprendedurismo, así como transferir conocimientos y habilidades. Mientras que algunos políticos continúan viendo a sus nacionales en el extranjero como una pérdida, cada vez son más los que reconocen que una diáspora comprometida puede ser un activo - o incluso un contrapeso a la emigración de personas cualificadas y de talento» (Newland y Plaza, 2013).

Afortunadamente está cambiando la valoración de lo que significa ir a vivir y trabajar en el extranjero. Se está extendiendo poco a poco la idea de que la internacionalización del talento, trabajar en otros países, es algo positivo: para los propios profesionales, en primer lugar, por todo lo que aporta a su formación, sus conocimientos y capacidades; y también para la economía, por el activo que el talento internacional puede suponer como apoyo a la internacionalización de una economía.

Esta nueva visión se reflejaba por ejemplo en un artículo publicado por El País el pasado mes de diciembre de 2018. En él se aludía de forma clara a la valoración positiva que para los profesionales tiene una experiencia de trabajo en el exterior: «Esto es algo incuestionable en las escuelas de negocios. Los tres centros consultados para este artículo, IE, IESE y Esade, con un elevado porcentaje de alumnos extranjeros en sus aulas, indican que actualmente los directivos ya han asumido que necesitan la experiencia internacional para progresar en su carrera. Todos ellos se plantean trabajar al menos durante tres o cuatro años fuera de nuestras fronteras. «Ha cambiado el paradigma», asegura María Obiols, directora de carreras profesionales de Esade, «hoy se persigue lo que se conoce como el triple salto, que implica un movimiento geográfico, de sector y de función» (Sánchez-Silva, 2018).

\section{La experiencia internacional como vía para reforzar el talento de los profesionales}

La experiencia laboral en otros países puede aportar muchos activos a un profesional: desarrollo de la capacidad de trabajo en equipos multiculturales, conocimiento de otros métodos y culturas empresariales, capacidades para negociación internacional e intercultural, dominio de idiomas, etc. Para todos los profesionales, y en particular para los jóvenes, una experiencia profesional internacional es una oportunidad de desarrollar de forma notable su talento. (Dejamos a un lado otro aspecto que puede ser también muy importante: el enriquecimiento personal y vital).

Sobre las ventajas que tiene para la capacidad profesional de una persona el hecho de vivir en el extranjero, se publicó en 2018 un artículo en Harvard Business Review (Adam et al., 2018) que recoge los resultados de una serie de estudios realizados por varios profesores de escuelas de negocios de Estados Unidos. Las conclusiones son bastante terminantes: vivir, trabajar y estudiar en el extranjero refuerza las capacidades psicológicas de una persona, promueve su creatividad, y contribuye al éxito en sus carreras profesionales.

Muchos españoles que, por ejemplo, han ido a trabajar a países del norte de Europa o Norteamérica, han podido conocer y aprender una cultura de trabajo que es más práctica que la española, con una organización menos jerárquica, un espíritu más igualitario que fomenta la participación de los trabajadores. La comunicación es más directa, la gente dice lo que piensa sin el respeto o temor a los «jefes» que existe en España. Es una cultura de trabajo que favorece la innovación y la colaboración, una cultura de la que España está necesitada.

Como señala una psicóloga en un artículo en El País, que se fue al Reino Unido, «Mi etapa como emigrante es lo peor que me pasó en la vida, porque hubo momentos muy duros. Pero también ha sido lo mejor. Hoy no sería la que soy si no me hubiese ido» (Doncel, 2017).

Hay que tener en cuenta que, como ha sucedido en muchos países que han tenido fuertes flujos migratorios al exterior, una buena parte de estos profesionales regresará en el futuro a su país de origen y traerá consigo estos conocimientos y esta nueva mentalidad.

Existen indicios de que esta valoración positiva sobre la experiencia en el extranjero se está 


\section{CUADRO 1}

\section{¿CUÁLES FUERON SUS RAZONES PARA SALIR DE ESPAÑA?}

(En \%, respuesta múltiple)

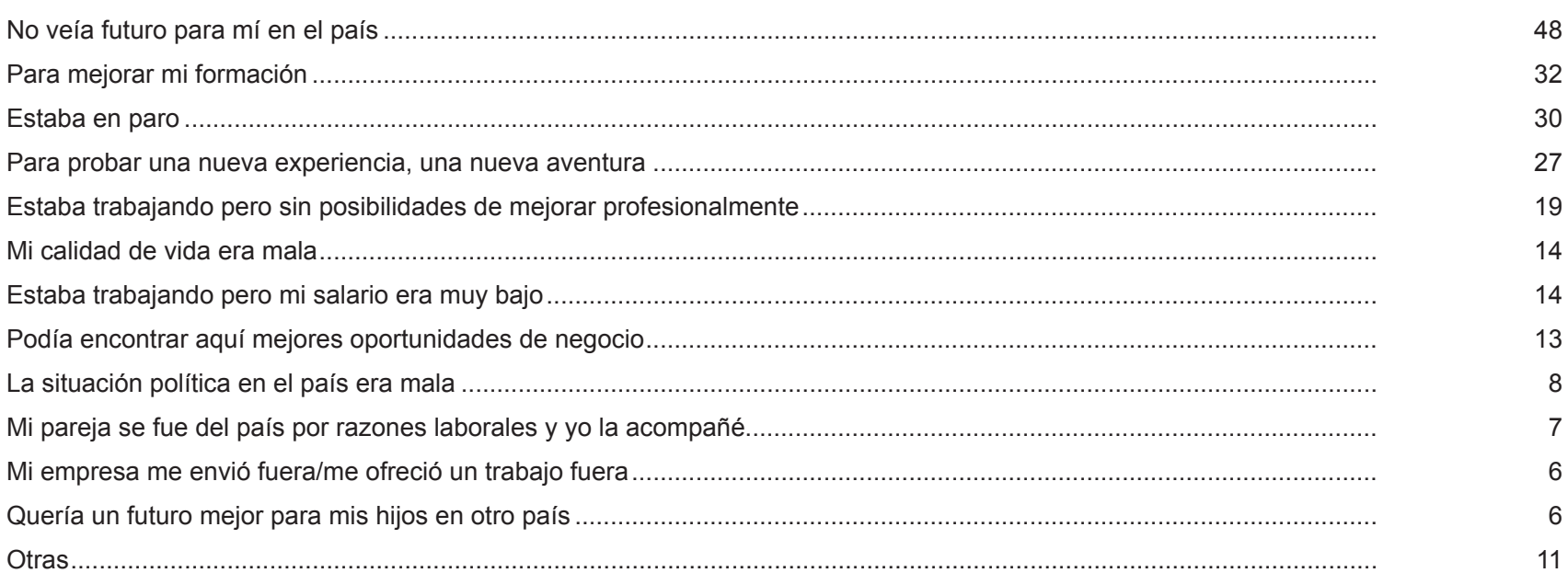

FUENTE: González Enríquez y Martínez Romera (2014).

extendiendo entre la sociedad española, en especial entre los jóvenes. Es demasiado pronto para disponer de un conjunto de trabajos que aporten un conocimiento a fondo de la experiencia de los profesionales españoles que salieron al exterior a partir de 2008. Pero ya se ha publicado algún trabajo que ofrece datos significativos. Investigadores del Real Instituto Elcano realizaron en 2013 , en el marco de una iniciativa conjunta con otros centros europeos de investigación, una encuesta entre españoles que habían emigrado al exterior a partir de 2007.

En el Cuadro 1 se recogen las razones alegadas por los encuestados para salir de España. Los que indican que se encontraban en paro representan solo el $30 \%$. La razón más aludida para marcharse es la de «no veía futuro para mí en el país», con un $48 \%$ de los encuestados. Pero un destacable $32 \%$ señala como razón «para mejorar mi formación», y un 27 \% «para probar una nueva experiencia, una nueva aventura» (para valorar bien estos porcentajes hay que tener en cuenta que se admitían respuestas múltiples).
Vemos por tanto que aunque las motivaciones que podríamos calificar como «negativas» (estar en paro, ver un futuro incierto) eran desde luego importantes, también lo eran las que podríamos calificar como «positivas» (mejorar la formación, probar nuevas experiencias), lo que refleja ese cambio en cómo se percibe la marcha al extranjero.

El Cuadro 2 recoge la distribución por estudios de los que respondieron a la encuesta. Hay que destacar que, según la nota de prensa que publicó el Real Instituto en su web sobre la encuesta, «el $91 \%$ de los emigrantes recientes tiene un título universitario», aunque de los diversos textos publicados por el Real Instituto Elcano sobre la encuesta parece deducirse que el dato se refiere a los que respondieron la encuesta. En todo caso, se trata de un dato que marca la gran diferencia existente entre la reciente ola de emigración española y la gran ola de emigración que se produjo en los años sesenta y setenta del siglo $X X$. Entre estos emigrantes con titulación superior, el mayor porcentaje correspondía a «ingeniería» (un 
CUADRO 2

\section{DISTRIBUCIÓN POR ESTUDIOS SEGÚN ENCUESTA}

(En \%)

Tipos de estudios

Porcentaje sobre el total con educación superior

Ciencias Sociales

15

Economía y Dirección de Empresas ........................................

Ingeniería..............................................................

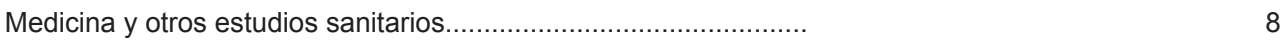

Humanidades y Educación .................................................. 11

Matemáticas y Ciencias naturales ................................... 10

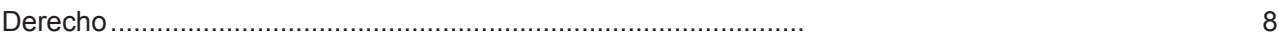

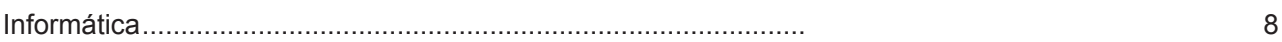

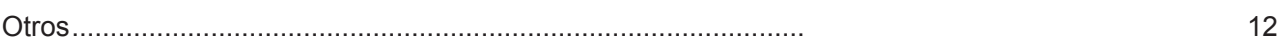

FUENTE: González Enríquez y Martínez Romera (2014).

$31 \%$ ), seguidos de «economía y dirección de empresas» (un $17 \%$ ) y «ciencias sociales» (un $15 \%$ ).

En todo caso, el dato clave es que en la emigración reciente de españoles tienen un peso muy importante los profesionales con estudios superiores, de los que cabe esperar una mayor capacidad de influencia en la actuación de las empresas y las instituciones en las que trabajen $\mathrm{y}$, por tanto, una mayor potencialidad para ayudar a la proyección exterior de la economía española.

\section{La diáspora de profesionales como activo para sus países de origen}

Las diásporas pueden ser para sus países de origen una importante fuente de beneficios, en primer lugar como un activo que puede favorecer su internacionalización a través de diversos canales. Como resume Ricardo Hausmann, «la diáspora de un país, y las diásporas que este alberga, pueden ser un enorme activo para su desarrollo (...) Son un canal a través del cual pueden fluir no solo dinero sino también conocimientos, y son una fuente potencial de oportunidades para el comercio, la inversión, la innovación y las redes de profesionales» (Hausmann, 2015).

Los profesionales de un país en el extranjero pueden contribuir al desarrollo de negocios con su país de origen. Estos profesionales, asentados en sus países de emigración, cuentan con conocimientos y contactos en este, están bien situados para colaborar con empresas de su país de residencia para desarrollar su actividad en su país de origen. Igualmente, pueden ayudar a las empresas de su país de origen para desarrollar negocios en sus países de residencia. Pensemos, a modo de referencia, en el papel clave que han tenido las diásporas china e india en el proceso de internacionalización de las dos grandes potencias asiáticas. Las diásporas china e india han actuado como intermediarios de negocios, desempeñando un amplio abanico de funciones, entre los países en los que viven y China e India.

La conciencia de estas ventajas ha determinado que se hayan ido creando una serie de organizaciones dirigidas hacia la diáspora profesional, en una serie de países.

El papel de estas organizaciones se puede analizar desde una doble perspectiva: 
1) Las ventajas que pueden suponer para los propios miembros de la diáspora.

2) Las ventajas que una diáspora profesional puede aportar a sus países de origen y, en especial, a la internacionalización de su economía y de sus empresas.

En cuanto al primer tipo de ventajas, aquellas que beneficiarían directamente a los miembros de la diáspora, se pueden destacar las siguientes:

1) La creación de canales de networking entre los miembros de la diáspora, canales que facilitan contactos con empresas y entre profesionales. Estos canales constituyen en última instancia un medio para potenciar el progreso profesional, detectar oportunidades de negocio, obtener información, etcétera.

2) La implantación de actividades de mentoring y apoyo para nuevos llegados a un país extranjero, que pueden facilitar una mejor integración de los mismos.

3) El establecimiento de mecanismos de ayuda para los que en un momento determinado deseen retornar a sus países de origen. Es decir, instrumentos que apoyen a los profesionales que quieren retornar, con información, ayuda para gestiones administrativas, ayuda para encontrar empleo.

Sobre el segundo tipo de ventajas, para los países de origen, se pueden destacar las siguientes:

1) Los profesionales en el exterior pueden servir para canalizar tecnologías, inversiones y, en general, negocios hacia sus países de origen. En algunos casos pueden ser los mismos profesionales los que lleven a cabo estas inversiones, a través de empresas en las que detentan una parte importante de propiedad. En otros casos pueden servir para favorecer la canalización de inversiones de las empresas en las que trabajan. Refiriéndose al caso de India, Devane recoge en su artículo (Devane, 2006) unas declaraciones de Alok Aggarwal, un alto ejecutivo de origen indio que ha ocupado posiciones de importancia en IBM, entre otras empresas: «Los expatriados han proporcionado valiosos vínculos con los mercados extranjeros, ayudaron a las empresas indias a absorber prácticas técnicas y gerenciales y a establecer contactos con clientes extranjeros. Por ejemplo, algunos indios que habían emigrado para trabajar en empresas estadounidenses en la década de los años ochenta han ayudado a compradores estadounidenses a encontrar proveedores en India (...) en diversos casos el impulso inicial para la subcontratación provino de empleados de origen indio. La mayoría, si no todas, de las filiales de Estados Unidos en India están dirigidas o cuentan con empleados de origen indio». Devane destaca cómo los ejecutivos de alto nivel de origen emigrante pueden desempeñar un papel para orientar la actividad de sus empresas hacia sus países de origen: «Los actores clave son los expatriados que se han convertido en altos ejecutivos de empresas relevantes. Teniendo en cuenta que la mayoría de las grandes empresas están considerando la subcontratación internacional de servicios empresariales facilitados a través de Internet, el papel clave para estos expatriados bien posicionados es crear conciencia en sus empresas de que sus países de origen pueden ser candidatos para este outsourcing».

2) Los profesionales de la diáspora pueden ser una fuente de información sobre la percepción que existe sobre su país de origen en sus países de residencia, pueden facilitar inteligencia, inputs de gran utilidad tanto para las Administraciones de sus países de origen como para empresas y organizaciones empresariales. Los profesionales pueden ser una fuente de información para la realización de estudios de mercado, el diseño de campañas de publicidad, identificación de socios, etcétera. Su posición ofrece unas ventajas únicas: conocen bien el país en el que residen y al mismo tiempo conocen el país del que son originarios.

3) Colaboración para el desarrollo de negocios con empresas e instituciones de sus países de origen. Esta es quizás la vía en la que los profesionales en el exterior pueden contribuir de manera más directa a promover la internacionalización de sus países de origen. Las empresas de estos pueden necesitar en un determinado momento servicios profesionales, contactos, información, realización de 
estudios, para su trabajo en un mercado determinado. Los profesionales en el exterior pueden proveer estos servicios, aprovechando el activo que supone el conocimiento del mercado en el que residen y el conocimiento de la cultura y métodos de trabajo del país del que son originarios. La colaboración con empresas puede extenderse a diversos campos. Un profesional puede actuar como asesor o agente de una empresa de su país originario. Puede actuar igualmente como intermediario para localizar clientes, socios, suministradores, en su país de residencia. Esto se aplica de forma especial, aunque ni mucho menos exclusiva, a profesionales que trabajan en el sector de servicios a empresas. Una empresa española, por ejemplo, que necesite un abogado en un determinado país, puede encontrar en un abogado español implantado en este país unas claras ventajas: el abogado conoce el funcionamiento del marco legal en el país en el que reside, y al mismo tiempo conoce la cultura española, los métodos de trabajo de las empresas españolas, y lo más probable es que conserve un conocimiento del funcionamiento del marco legal en España.

Las ventajas de esta asociación son claras: los profesionales en el exterior conocen el país en el que viven (y el sector en el que trabajan), y al mismo tiempo conocen España, su cultura. Están bien posicionados por su carácter bicultural para trabajar como apoyo para las empresas españolas, para hacer de "puentes» de estas en mercados que les son desconocidos. Se trata de una asociación que beneficia a los dos lados: a las empresas y a los profesionales (Fanjul, 2017). Un factor clave para desempeñar este papel de intermediación entre dos países, y dos culturas es, como hemos indicado, el conocimiento que los profesionales de la diáspora tienen de estas. Estos profesionales conocen las lenguas de los dos países, sus costumbres, sus normas sociales, sus culturas. Los profesionales de un cierto nivel de cualificación conocen sus economías, prácticas empresariales, instituciones. Y tienen contactos en los dos países. Es frecuente que entre los profesionales de un país exista una cierta desconfianza hacia la realización de negocios en otros países, sobre los que tienen un conocimiento escaso. Los profesionales de la diáspora pueden facilitar la construcción de un marco de relaciones que permita superar, al menos parcialmente, esta desconfianza. "Con una comprensión profunda de las normas culturales y sociales, de las distintas culturas empresariales y los idiomas locales, los profesionales de la diáspora construyen vínculos de confianza y abren oportunidades a menudo cerradas o desconocidas para otros empresarios. Estas redes también se pueden utilizar para obtener conocimiento sobre oportunidades de mercado» (Dutia, 2012).

4) Los profesionales pueden retornar a su país de origen, finalmente. Llevarán a sus países de origen los conocimientos que hayan adquirido, los contactos en los países en los que vivieron, el conocimiento de estos... Ricardo Hausmann (Hausmann, 2015) destaca por ejemplo cómo el sector tecnológico indio ha sido desarrollado sobre todo por emigrantes que retornaron al país.

Según señalan Newland y Plaza, «se ha acumulado la evidencia de una fuerte correlación entre la presencia de una diáspora residente en un país y la existencia de vínculos comerciales con el país de origen de la diáspora. Un estudio sobre el comercio de Canadá con 136 países en el período 1980-1992 mostraba que un aumento del $10 \%$ en la inmigración procedente de un determinado país estaba asociado con un aumento de un $1 \%$ en las exportaciones y un aumento del $3 \%$ en las importaciones con dicho país» (Newland y Plaza, 2013).

Newland y Plaza mencionan en su trabajo estudios similares, que muestran un fuerte vínculo entre la presencia de diásporas y un aumento del comercio con sus países de origen. Algo parecido se ha detectado en relación con los flujos de inversión, tanto inversión directa como inversión en cartera. Los países comercian e invierten más en los países de origen de las diásporas con las que cuentan. Asímismo, los países 
mejoran su eficiencia en la producción de bienes que los países de origen de sus inmigrantes son eficientes en producir.

Dany Bahar y Ernesto Talvi mencionan el caso de Vietnam: "La evidencia muestra que los emigrantes juegan un papel importante en el fomento del comercio a través de la creación de redes. Un ejemplo notable se puede encontrar en los patrones comerciales bilaterales entre Vietnam y Estados Unidos. En 1975, Estados Unidos evacuó a más de 100.000 refugiados de Vietnam del Sur y los llevó a Estados Unidos. Una vez en Estados Unidos, fue el Gobierno, no los propios refugiados, el que decidió su lugar de asentamiento. En esta época, y hasta mediados de la década de los años noventa, Estados Unidos mantuvo un embargo con Vietnam que imposibilitó el comercio entre los dos países (...) Tras el levantamiento del embargo, la diáspora vietnamita en Estados Unidos, que se había extendido por diferentes Estados en diferentes proporciones, desempeñó un papel en la reanudación del comercio bilateral con Vietnam. Los Estados que habían recibido más refugiados vietnamitas comerciaron más con Vietnam» (Bahar y Talvi, 2018).

Bahar y Talvi estudian en su artículo el papel de las diásporas en la difusión de conocimientos y habilidades. "La importancia de los emigrantes como impulsores de nuevos conocimientos que se traduce en una mayor productividad está bien documentada. El papel de los emigrantes en la difusión del conocimiento actúa en las dos direcciones. Por ejemplo, grandes empresas que contratan a emigrantes retornados tienen un mejor desempeño en términos de patentes. La emigración desempeña un papel en la mejora de la productividad a nivel industrial para los países tanto emisores como receptores de emigrantes».

Por su parte, Dutia destaca el caso de Silicon Valley. «Otro ejemplo bien conocido es el de los empresarios inmigrantes de Silicon Valley, que lideraron el fenómeno de las puntocoms en la década de los noventa al emprender negocios tecnológicos que generaron beneficios económicos y empleo en Estados Unidos y también crearon una red y negocios en sus países de origen. Hoy están contribuyendo a la creación de nuevos centros de tecnología y conocimientos en sus países de origen. A medida que estos empresarios colaboran con antiguos compañeros de clase y colegas en economías que habían estado poco activas, como India y China, proporcionan acceso a mercados y know-how que son críticos para el éxito en la economía global de nuestros días. Esto también es aplicable a los «inmigrantes» que trabajaron y estudiaron en Estados Unidos y el Reino Unido y han regresado a sus países de origen. Este grupo de expatriados de la diáspora aprovechan sus vínculos y conexiones en Estados Unidos y han estimulado las innovaciones tecnológicas y la actividad económica» (Dutia, 2012).

\section{Motivaciones de la diáspora}

Como hemos comentado, una serie de países, conscientes de la importancia que puede tener su diáspora de profesionales, han creado organizaciones con el fin de aprovechar sus ventajas.

La cuestión clave que cabe plantearse es: ¿por qué participarían los profesionales establecidos en el exterior en una organización promovida por su país de origen?

Existen tres motivaciones fundamentales para que los miembros de una diáspora estén dispuestos e interesados en participar en una organización de este tipo:

- En primer lugar, por su propio interés profesional. Como hemos indicado en el apartado anterior, los contactos con otros miembros de la diáspora, o con empresas e instituciones de su país de origen, pueden ser una fuente de negocios y de oportunidades profesionales. Una de las actividades principales que llevan a cabo las organizaciones creadas para atender y movilizar las diásporas es precisamente la celebración de encuentros, seminarios, eventos de networking, que faciliten el establecimiento de este tipo de contactos. Estas actividades pueden desarrollarse también no 
solo mediante actividades presenciales sino aprovechando las facilidades que ofrece las nuevas tecnologías para interactuar en Internet.

- En segundo lugar, muchos miembros de estas diásporas pueden pensar que en un futuro, más o menos lejano, tendrán interés en retornar a su país de origen. Pertenecer a una organización que tiene instrumentos para ayudarlos en el proceso de retorno es pues un incentivo para los profesionales en el exterior.

- En tercer lugar existe un aspecto difícilmente cuantificable pero que puede desempeñar también un papel importante. Se trata de lo que podríamos denominar el «vínculo afectivo» o "patriótico» con el país de origen. Muchos expatriados mantienen vínculos familiares y de otro tipo con sus países de origen. Sienten una vinculación afectiva con estos, y con frecuencia están dispuestos a llevar a cabo una actividad altruista, sin pedir una contrapartida, con el fin de apoyarlos. Muy unido a este factor se encontraría el deseo de mantener un contacto con el país de origen, por el vínculo afectivo mencionado anteriormente, la idea de que quizás en un futuro más o menos lejano el expatriado podrá desear retornar al mismo, porque tiene familia en él. No hay duda de que este vínculo afectivo o patriótico existe en numerosas personas, y constituye una de las bases para colaborar o participar en organizaciones dirigidas a la diáspora. Por este vínculo, la diáspora tiene una disposición para ayudar a mejorar su país de origen, contribuyendo a ello a través de diferentes medios. Dutia cuenta en su artículo el caso de Henry Ford. Consciente de los bajos niveles de desarrollo de Irlanda, Ford sintió una necesidad de aplicar sus conocimientos para revitalizar el área de Cork, muy deprimida económicamente, y de donde procedían sus orígenes familiares. «La decisión de elegir su tierra de procedencia como el país para expandir su empresa se basó en su deseo emocional de reconectarse y contribuir al desarrollo de su tierra ancestral. La historia de Ford ilustra la importancia que los vínculos de la diáspora pueden tener en el emprendimiento global» (Dutia, 2012).

\section{Estrategias sobre la diáspora}

Los Gobiernos de muchos países, en el marco de sus políticas de internacionalización, tienen un interés en desarrollar instrumentos que les permitan aprovechar el activo que representa la presencia en otros países de profesionales de su país.

Es importante definir con una cierta precisión el concepto de «diáspora», a los efectos de poder diseñar estrategias adecuadas: «la diáspora se define como una población distribuida por el extranjero que mantiene una afinidad con un hogar nacional y una comunidad a causa de un sentimiento común de ancestro, etnicidad e identificación» (Lynn-Ee Ho, Hickey y Yeoh, 2015).

Se trata por tanto de una definición relativamente amplia, y que consideramos adecuada a los objetos del presente artículo, ya que abre las puertas para considerar como integrantes de la diáspora a personas que, en el ejemplo de España, no tienen por qué ser necesariamente de nacionalidad española o de origen español, sino que pueden tener una afinidad con España por diversos motivos: laborales, familiares, etc.

El siguiente concepto que es importante definir es el de estrategias sobre la diáspora. De nuevo Lynn-Ee, Hickey y Yeoh recurren a una definición sencilla y de amplio alcance, susceptible de albergar una gran variedad de actuaciones: «las estrategias sobre la diáspora representan los medios por los que las nacionesEstado ejercen una acción extraterritorial para afirmar su influencia sobre las poblaciones de su diáspora». Es decir, las estrategias sobre la diáspora son «iniciativas encaminadas a movilizar los emigrantes con el propósito de favorecer el desarrollo económico y/o la construcción nacional».

Según Newland y Plaza, el diseño de una estrategia sobre la diáspora tiene una serie de ingredientes clave (Newland y Plaza, 2013):

1) Identificar y mapear la diáspora, es decir, identificar en dónde se encuentran los principales focos de presencia de la misma. Ello requiere diseñar un mecanismo que 
permita desarrollar una base de datos de profesionales en el exterior, que compatibilice el respeto a su privacidad con la obtención de una serie de datos que faciliten en un momento dado la identificación y el contacto con los miembros de la diáspora que sean más adecuados para una determinada finalidad. Lógicamente, es importante conocer cuál es la categoría del profesional (empleado o directivo de una empresa, funcionario, académico, etc.), su sector y su nivel profesional.

2) Instrumentar políticas y actividades que faciliten la involucración y movilización de la diáspora.

3) Coordinar la actuación de los diferentes organismos públicos cuya acción es relevante para la diáspora.

4) Establecer mecanismos de colaboración con entidades privadas o semiprivadas como Cámaras de Comercio, asociaciones empresariales, universidades, escuelas de negocios, etcétera.

5) Establecer instrumentos de comunicación que permitan llegar de forma efectiva a la diáspora. Estos instrumentos tienen que basarse en los tiempos actuales en Internet y en las redes sociales.

Otro aspecto importante que se trata en el trabajo de Newland y Plaza es la naturaleza de los actores que ejecutan las políticas sobre la diáspora. En este sentido puede decirse que hasta ahora ha habido un predominio de actores estatales, es decir de organizaciones impulsadas y creadas por las Administraciones públicas. Hay algunos países que incluso han establecido un ministerio para asuntos de la diáspora. En algunos casos se han articulado mecanismos de representación política para los miembros de la diáspora (Bahar y Talvi, 2018).

Un ejemplo de organización cuya finalidad específica es el aprovechamiento del activo que supone la diáspora es Advance, una organización de Australia cuyo objetivo, Connecting Globally Australians, es aprovechar el recurso que suponen los millones de australianos que hay por el mundo. Chile, por su parte, lanzó con una finalidad similar Chile Global, que se define (en su cuenta de Twitter) como una «Red de talentos en el exterior de Imagen de Chile, que agrupa a empresarios, estudiantes y profesionales chilenos y amigos de Chile, que viven en el extranjero».

Desde hace algún tiempo están adquiriendo una creciente importancia actores que no son de naturaleza pública, que surgen desde la sociedad civil, apoyados por fundaciones $u$ otras instituciones sin ánimo de lucro. En el caso de España cabe resaltar la actuación de la Fundación Rafael del Pino y, de modo especial, la Fundación Ramón Areces, que patrocina la red de asociaciones de científicos españoles en el extranjero. Algunas Cámaras de Comercio españolas en el exterior han desarrollado también iniciativas relacionadas con la diáspora. Instituciones educativas, como escuelas de negocio, cuentan igualmente con redes de antiguos alumnos, que pueden ser consideradas como organizaciones susceptibles de colaborar con iniciativas dirigidas a la diáspora profesional.

Han surgido de hecho asociaciones de profesionales españoles en algunos países. Probablemente los más avanzados en este aspecto son los científicos. Han surgido asociaciones de científicos españoles en numerosos países, que se han agrupado en RAICEX, Red de Asociaciones de Investigadores y Científicos Españoles en el Exterior, constituida en 2018 con el apoyo de la Fundación Ramón Areces.

Desde la perspectiva de España, la cuestión clave es: ¿cómo hacer para utilizar de forma efectiva este gran capital humano que supone la comunidad de profesionales españoles en el mundo? Como han hecho, entre otros, los australianos, es preciso crear un instrumento que facilite la conexión con estos profesionales. Este instrumento, u organización, que probablemente debería estar basado en una colaboración público-privada, debe en primer lugar establecer un sistema para identificar y ordenar a los profesionales.

En segundo lugar, esta organización debe actuar como una red de redes, promoviendo la comunicación con las redes que ya existen, y la intercomunicación entre estas.

En tercer lugar, debe servir como una plataforma de contacto para los profesionales y las redes en el 
exterior con empresas e instituciones españolas: muchos de los profesionales en el exterior llevan años fuera de España, y necesitan información y contactos sobre España, sus sectores y empresas. La organización debe ser un instrumento de conexión entre las empresas españolas y los profesionales $-\mathrm{y}$ sus redes- en el exterior.

Finalmente, una organización de este tipo también debería servir para apoyar a los profesionales en el exterior: suministrándoles información sobre España, dando orientación y apoyo a aquellos que tengan interés en regresar a España, etc.

Existen dos grandes modelos en cuanto a la incorporación a una organización de este tipo, dirigida a aprovechar el capital humano de los profesionales en el exterior. Por un lado, puede ser una organización «abierta», en la que se pueden incorporar todas aquellas personas que lo deseen. Lógicamente, deben cumplir unos mínimos requisitos en cuanto a su perfil profesional: ser directivos de empresas, profesionales, funcionarios de nivel superior. Probablemente pueda ser complicado establecer unos criterios claros a este respecto. El criterio último es que por su posición profesional puedan ser de utilidad a la internacionalización de la economía del país, en un sentido muy amplio.

Un matiz importante es que, como hemos comentado anteriormente en este artículo, una organización de este tipo esté abierta no solo a personas que son nacionales $u$ originarias del país, sino también a personas que puedan tener una afinidad con el mismo, por razones familiares o profesionales (trabajan o tienen interés en desarrollar actividades profesionales o de negocios con el país). La organización australiana Advance sería un buen ejemplo de este tipo de modelo.

El otro modelo es «cerrado». No contempla la incorporación abierta de profesionales, sino que esta se produce por una invitación, personal e intransferible. Se trata de un modelo más selectivo, en el que se pretende contar con un grupo relativamente reducido de miembros, que ocupen puestos de relevancia en el mundo profesional. Un ejemplo muy significativo sería GlobalScot, que se define como una «red mundial de emprendedores y líderes empresariales dedicados a apoyar a las empresas de Escocia». GlobalScot ofrece a las empresas escocesas la posibilidad de contar con la asistencia de una red de empresarios y profesionales de alto nivel, que les asesoran y ayudan en sus labores de internacionalización. La red cuenta con más de 600 GlobalScots, que actúan en unos 50 países, $y$, aparte de facilitar el contacto entre empresas escocesas y miembros de la red, organiza regularmente eventos y encuentros empresariales.

En este artículo he tratado la diáspora de profesionales como un activo de gran potencial para apoyar la internacionalización de una economía. No he tratado otra dimensión del fenómeno de las diásporas que puede tener también relevancia, al que voy a hacer una breve mención. Me refiero a la presencia en un país de una diáspora de profesionales que tienen un origen extranjero, lo que en inglés se llama inbound diasporas. Proceden de la inmigración que ha recibido el país en el pasado. A esta diáspora de origen extranjero en un país se le pueden aplicar muchas de las consideraciones que hemos realizado sobre la diáspora de profesionales de ese país que se halla en el extranjero. Estos profesionales pueden cumplir las funciones de intermediación, establecimiento de contactos, promoción de negocios, generación de confianza, con sus países de origen. Estos profesionales tendrán normalmente familiares y contactos en dichos países, sentirán también un vínculo afectivo con ellos. Y muchos de ellos serán conscientes de que cuentan con un activo, el conocimiento y los contactos en su país de origen y en su país de residencia actual, que puede resultarles de gran utilidad para su desarrollo profesional y para los negocios.

Javier Santiso es probablemente uno de los primeros autores que prestó atención en España a este tema. En un artículo publicado en 2012, y titulado de forma significativa Los Nuevos Argonautas Latinos: ¿Podrían ayudar en la internacionalización de España? Santiso se refirió en concreto al papel que pueden desempeñar 
los profesionales de origen latinoamericano que residen en España como impulsores de las relaciones económicas con los países latinoamericanos: «Estas diásporas latinas, en particular las asentadas en España, podrían ayudar a tejer vínculos con sus países de origen (...) Igualmente, las diásporas latinas, en particular en multinacionales europeas, podrían ser buenos 'embajadores' para movilizar e incentivar la ubicación de sedes para América Latina de estas multinacionales. Dicho de otra manera, España debería considerar la manera estratégica de movilizar este tipo de diáspora para incentivar el asentamiento de sedes corporativas, centros de $I+D$, etc. en la península. Los latinoamericanos ubicados en España así como los ubicados en Europa, pueden ser unos activos muy potentes en una estrategia sistémica para fomentar esta estrategia. Sin embargo, España carece de un 'Real Consejo Internacional', el cual podría estar vinculado con la Casa Real, con el Príncipe por ejemplo, y con Moncloa. Tendría así un rango y una categoría simbólica importante y a su vez una operatividad que se podría combinar con el ICEX-Invest in Spain» (Santiso, 2012).

En suma, un país, como España, tiene dos importante activos que pueden serle de gran utilidad en su internacionalización: por un lado, los profesionales españoles que viven en otros países, y cuyo número y relevancia ha crecido de manera espectacular en la última década; $y$, por otro, los profesionales de origen inmigrante que viven en España.

\section{Referencias bibliográficas}

Adam, H., Obodaru, O., Lu, J., Maddux, W., \& Galinsky, A. (2018). How Living Abroad Helps You Develop a Clearer Sense of Self. Harvard Business Review. https://hbr. org/2018/05/how-living-abroad-helps-you-develop-aclearer-sense-of-self

Bahar, D., \& Talvi, E. (2018). How can developing countries use their diaspora capital? Brookings Institution. https:// www.brookings.edu/research/how-can-developing-countries-use-their-diaspora-capital/

Cheng, J. (2016). Engaging Diasporas: The case of Australia and other key countries. Australian Council of
Learned Academies. https://acola.org.au/wp/PDF/SAF11/ Cheng\%20Jonathan.pdf

Devane, R. (2006). The Dynamics of Diaspora Networks: Lessons of Experience. In Y. Kuznetsov (Ed.), Diaspora Networks and the International Migration of Skills: how countries can draw on their talent abroad. World Bank Institute. http://documents.worldbank.org/curated/ en/465841468313860840/pdf/365850PAPER0Diaspora01OFFICIALOUSE0ONLY1.pdf

Doncel, L. (2017). El talento quiere volver a casa. El País. https://elpais.com/economia/2017/05/20/actualidad/1495286170_018584.html

Dutia, S. (2012). Diaspora Networks: A New Impetus to Drive Entrepreneurship. Innovations: Technology, Governance, Globalization, 7(1), 65-72. https://papers.ssrn.com/sol3/ papers.cfm?abstract_id=2373984

Elo, M. (2014). Diaspora networks in international business and transnational entrepreneurship - A literature review. Center for Transnational Studies of the Universities of Bremen and Oldenburg. https://www.researchgate.net/ publication/267863088 Diaspora Networks in International_Business_and_Transnational_Entrepreneurship_A_Literature_Review

Fanjul, E. (2013). Negociación intercultural. Smashwords/lberglobal.

Fanjul, E. (2016). El déficit en idiomas como barrera a la internacionalización. Real Instituto Elcano Blog. https://blog. realinstitutoelcano.org/deficit-idiomas-barrera-internacionalizacion/

Fanjul, E. (2017a). Un activo para la internacionalización: la diáspora de profesionales españoles. Real Instituto Elcano Blog. https://blog.realinstitutoelcano.org/activo-internacionalizacion-diaspora-profesionales-espanoles/

Fanjul, E. (2017b). ¿Tiene sentido incentivar el retorno del talento que se fue al exterior? Real Instituto Elcano Blog. https://blog.realinstitutoelcano.org/incentivar-retorno-talento-exterior/

Fanjul, E. (2018a). Internacionalización del talento: por un cambio de actitud. Real Instituto Elcano Blog. https:// blog.realinstitutoelcano.org/internacionalizacion-del-talento-por-un-cambio-de-actitud/

Fanjul, E. (2018b). Un instrumento para la diáspora profesional española. Real Instituto Elcano Blog. https://blog.realinstitutoelcano.org/un-instrumento-para-la-diaspora-profesional-espanola/

González Enríquez, C., y Martínez Romera, J.P. (2014). España: Emigración de los españoles autóctonos durante la crisis. Real Instituto Elcano. http://www.realinstitutoelcano.org/ wps/portal/rielcano_es/contenido?WCM_GLOBAL_CONTEXT=/elcano/elcano_es/zonas_es/demografia+y+polacibon/gonzalezenriquez-martinezromera-espana-em- 
igracion-espanoles-crisis-spain-migration-crisis http:// www.realinstitutoelcano.org/wps/portal/rielcano_es/prensavista?WCM_GLOBAL_CONTEXT=/elcano/elcano_es/ prensa/notas/encuesta-emigracion-espanoles-crisis

Hausmann, R. (2015). The Diaspora Goldmine. Project Syndicate-The World's Opinion, 25. https://www.project-syndicate.org/commentary/remittances-not-only-benefit-to-homeland-economies-by-ricardo-hausmann-2015-06?barrier=accesspaylog

InterNations (2016). Expat Insider 2016: Three Years of Insights. https://www.internations.org/expat-insider/2016/

Kuznetsov, Y. (2006). Diaspora Networks and the International Migration of Skills: How Countries Can Draw on their Talent Abroad. WBI Development Studies. https://openknowledge.worldbank.org/handle/10986/7008

Lynn-Ee Ho, E., Hickey, M., \& Yeoh, B. (2015). New Research Directions and Critical Perspectives on Diaspora Strategies. Geoforum, 59, 153-158. http://scholarbank.nus.edu. sg/bitstream/10635/123071/1/2015-Special_issue_introduction_new_research-postprint.pdf

Manzano, C. (2013). Es hora de que los españoles salgan de su aislamiento. Política Exterior, 27(152), 98-106. http:// www.politicaexterior.com/articulos/politica-exterior/eshora-de-que-los-espanoles-salgan-de-su-aislamiento/
Newland, K., \& Plaza, S. (2013). What We Know about Diasporas and Economic Development. Migration Policy Institute. https:// www.migrationpolicy.org/research/what-we-know-about-diasporas-and-economic-development

Niebhur, A. (2006). Migration and Innovation. Does Cultural Diversity Matter for Regional R\&D Activity? IAB Discussion Paper. http://doku.iab.de/discussionpapers/2006/dp1406.pdf

Quintanilla, J., Sánchez-Mangas, R., y Susaeta, L. (2010). Políticas y prácticas de recursos humanos en el contexto organizativo de la empresa multinacional: Un análisis comparativo internacional. Fundación BBVA-IESE. https://www.fbbva.es/publicaciones/politicas-y-practicas-de-recursos-humanos-en-el-contexto-organizativo-de-la-empresa-multinacional/

Sánchez-Silva, C. (2018). Directivos españoles que mandan por el mundo. El País. https://elpais.com/economia/2018/12/07/ actualidad/1544177391_973994.html

Santiso, J. (2012). Los Nuevos Argonautas Latinos: ¿Podrían ayudar en la internacionalización de España? Anuario de la Internacionalización de la Empresa Española. Círculo de Empresarios. https://circulodeempresarios.org/app/uploads/2016/03/4-cap.pdf

Toca, G. (2017). Depende: talento español en el extranjero. Esglobal. https://www.esglobal.org/depende-talento-espanol-extranjero/ 